\title{
A2241: CLUSTERS WITH HEAD-TAILS AT X-RAYS
}

\section{NORCI}

Dunsink Observatory

Castleknock, Dublin 15, Ireland

L. FERETTI

Istituto di Radio Astronomia

Via Gobetti 101, I-40129 Bologna, Italy

\author{
AND \\ E.J.A. MEURS \\ Dunsink Observatory \\ Castleknock, Dublin 15, Ireland
}

\begin{abstract}
A ROSAT PSPC image of the galaxy cluster Abell 2241 has been obtained, showing X-ray emission from the intracluster medium and from individual objects. The brightness distribution of the cluster gas is used to assess the physical conditions at the location of two tailed radio galaxies (in A2241E and A2241W, at different redshifts). Together with radio and $\mathrm{X}$-ray information on the two galaxies themselves the results are of relevance to the question of energy equipartition in radio sources.
\end{abstract}

\section{Background}

A cluster intergalactic medium plays an important role in determining the morphology and evolution of radio sources. The external gas can interact with a radio source in different ways: confining the source, modifying the source morphology via ram-pressure and possibly feeding the active nucleus.

We have obtained ROSAT X-ray data of the region of the cluster Abell 2241, which was previously studied by Bijleveld \& Valentijn (1982). A2241 was originally classified as an irregular galaxy cluster, until redshift measurements showed it to consist of two separate clusters projected onto each other: A2241W and A2241E, located at redshifts of 0.0635 and 0.1021 , respectively. 
According to the radio data obtained with the Westerbork Synthesis Radio Telescope and presented by Bijleveld \& Valentijn (1982), both clusters contain some radio emitting galaxies, with most of them showing extended structure. In particular, both clusters contain a radio galaxy characterized by a tailed radio structure: $1657+32$ in $\mathrm{A} 2241 \mathrm{~W}$ and $1658+32$ in A2241E. High-resolution VLA radio data for these radio galaxies are reported by Fanti et al. (1987) and references therein. Referring to Einstein data, Bijleveld \& Valentijn detected X-ray emission only from A2241E.

\section{New X-ray data}

We obtained observations of the region of the A2241 clusters both with the PSPC and HRI detectors of the ROSAT satellite, in order to get information both on the large scale X-ray emission and on the pointlike sources. The HRI observation resulted however only in a very short, aborted exposure of ca. 3000 seconds. Maps of the X-ray brightness distribution were produced by binning the photon events in a two dimensional grid and then smoothing with a gaussian filter. The emission around the centre of A2241E covers a large area, whereas a smaller clump is seen at the location of A2241W. Both clusters exhibit extended X-ray emission in this observation.

The central, strongest source coincides with the $\mathrm{cD}$ galaxy in $\mathrm{A} 2241 \mathrm{E}$, a blob just East of this source is associated with the A2241E head-tail galaxy. Another extended blob, to the SW, corresponds to the A2241W head-tail galaxy; the $\mathrm{cD}$ galaxy in this cluster appears not detected. Two moderately strong point sources, $\mathrm{E}$ and $\mathrm{W}$ of the central strong source, coincide with a star and a QSO, respectively, and are used for astrometric calibration of the X-ray field.

The HRI exposure detects the central strong source and the QSO to the W. Two other sources are seen in addition.

\section{Outlook}

After complete analysis of the PSPC field, the combination of X-ray and radio data will be used to investigate the interaction between radio sources and intracluster gas. A comparison of the equipartition pressures determined from the radio observations and the thermal pressures in the $\mathrm{X}$-ray gas is here of particular interest (see Feretti et al. 1992).

\section{References}

Bijleveld, W. and Valentijn, E. (1982) $A \mho A A 111,50$

Fanti, C., Fanti, R., de Ruiter, H. and Parma, P. (1987) $A \& A S$ 69, 57

Feretti, L., Perola, G.C. and Fanti, R. (1992) $A \mathcal{B} A$ 265, 9 